\title{
IGUAL, IGUALES E IGUALITOS. DIMINUTIVOS EN EL HABLA DE CARACAS
}

\author{
Irania MALAVER \\ Universidad Central de Venezuela
}

\section{RESUMEN}

El objetivo del presente artículo es estudiar el uso del sufijo diminutivo -ito con la partícula igual en el habla de Caracas; a partir de la descripción de las funciones comparativas y discursivas de igual, se analizan las funciones de las formas igualito/igualitos, igualita/igualitas. Como adjetivo y adverbio comparativo de manera, el diminutivo intensifica la noción comparada; del mismo modo, como marcador de discurso intensifica el distanciamiento que expresa igual, cuando se emplea con valor concesivo, por lo que el diminutivo se extiende también a nivel de discurso y muestra la misma polifuncionalidad que la forma base. El estudio se basa en el Corpus sociolingüistico PRESEEA-Caracas 2004-2010, del que se analizaron todos los casos de igual y sus variantes con el sufijo diminutivo: la frecuencia, las funciones y su empleo según el sexo, la edad y el nivel de estudios de los hablantes. Se constata, en primer lugar, que igual y las formas diminutivas alternan en los usos comparativos y discursivos: en las construcciones comparativas hay mayor ocurrencia de igual y en las construcciones discursivas, el diminutivo alcanza un $49 \%$ de los casos. En segundo lugar, la forma masculina singular igualito es la de mayor frecuencia y se emplea principalmente como marcador de distanciamiento. En tercer lugar, los factores sociales no influyen en el empleo del diminutivo por igual, si bien hay una frecuencia un poco mayor entre las mujeres. Se confirma que el uso del diminutivo con bases adverbiales es característico del habla de Caracas.

PALABRAS CLAVE: corpus PRESEEA-Caracas, diminutivo, gramaticalización, polifuncionalidad.

\section{ABSTRACT}

The aim of the present article is to study the use of the diminutive suffix -ito with the igual particle in the speech of Caracas; from the description of the comparative and discursive functions of igual, the functions of the forms with diminutive igualito / igualitos, igualita/igualitas are analyzed. As an adjective and comparative adverb in a way, the diminutive intensifies the comparative notion; in the same way, as a discourse marker it intensifies the distancing that it expresses, 
when it is used with a concessive value, so the diminutive also extends to the level of discourse and shows the same polyfunctionality as the base form. The study is based on the sociolinguistic Corpus PRESEEA-Caracas 2004-2010, from which all the cases of equals and their variants with the diminutive suffix were analyzed: frequency, functions and their use according to sex, age and level of Studies of speakers. It is noted, first, that igual and diminutive forms alternate in comparative and discursive uses: in comparative constructions there is greater occurrence of igual and in the discussion constructions, the diminutive reaches $49 \%$ of cases. Secondly, the singular masculine form igualito is the most frequent and is used mainly as a distancing marker. Third, social factors do not influence the use of the diminutive, although there is a slightly higher frequency among women. It is confirmed that the use of the diminutive with adverbial bases is characteristic of the speech of Caracas.

KEYWORDS: corpus PRESEEA-Caracas, diminutive, grammaticalization, multifunctionality.

Fecha de recepción: 05/09/2017

Fecha de aceptación: 06/10/2017

Fecha de la versión definitiva: 13/01/2018

\section{Objetivos ${ }^{1}$}

El español venezolano se caracteriza por el empleo notorio de diminutivos en sustantivos (chamito, ajito, reunioncita, facturita), en adjetivos como altico, calladita y en adverbios como cerquita, lejito, clarito, tempranito y ahorita (cf. Malaver 2017). También destacan diminutivos como igualitas, igualitos oigualito que, al tratarse de la partícula polifuncional igual, pertenecen a distintas categorías; así en (1) el diminutivo es un adjetivo y en (2) un adverbio:

(1) I: cuando se acerca así que lo ve $<$ cita $>$ ¡Dios mío pero ese muchacho es [igualito] a mi mamá! </cita $>$ mira / muchacho / yo regresé a la tierra otra vez / (CARA H22_050) ${ }^{2}$

(2) y me iba a a hablar con ella / entonces claro [igualito] me pasó en Maracaibo / (CARA_M33_108)

${ }^{1}$ Esta investigación concluyó durante la estancia de investigación realizada en abril-junio de 2017 gracias a la beca Gíner de los Ríos que otorga la Universidad de Alcalá. Agradezco a los colegas del Departamento de Filología, Comunicación y Documentación, miembros del grupo PRESEEA-Alcalá de Henares.

${ }^{2}$ Los ejemplos extraídos del PRESEEA-Caracas tienen un código entre paréntesis que indica el sexo (hombre, mujer), la edad (grupo 1: 20-34 años; grupo 2: entre 34-55 años; grupo 3: 55 años y más) y el grado de instrucción: 1 (estudios primarios), 2 (estudios secundarios), 3 (estudios universitarios). Las letras CARA corresponden a la identificación de la ciudad. 
Y, además, puede aparecer en ejemplos como (3), en los que la forma en diminutivo es un marcador de discurso:

(3) no fue directamente de que no soportó la operación como tal / sino que fue que hubo algo más / pero / bueno / como en todos lados / nada se supo / después todo se sabe / [igualito] nos enteramos (CARA_M11_009)

Como se aprecia, el diminutivo posee distintas funciones y categorías. El objetivo de este estudio es analizar el fenómeno de diminutivización de igual en el habla de Caracas; a partir de la descripción de las funciones de igual, se analizan las funciones de las formas con el sufijo diminutivo y el modo en que estas formas, especialmente igualito, se relacionan con el proceso de gramaticalización de igual como marcador de discurso. Además de describir las funciones semántico-pragmáticas, se presenta un análisis cuantitativo de los usos del diminutivo obtenidos del Corpus sociolingüístico PRESEEA-Caracas 2004-2010².

Se ha dividido el presente artículo en cinco secciones además de los objetivos; en la segunda sección se presentan los aspectos teóricos que permiten describir las funciones sintáctico-pragmáticas de igual; la tercera sección se destina a la descripción del diminutivo y a las variantes igualitas, igualita, igualitos e igualito; el corpus de estudio y los criterios para la clasificación y descripción de los casos se exponen en la cuarta sección. Los resultados se discuten en la quinta sección y por último se presentan las conclusiones en la sexta sección.

\section{EL PROBLEMA DE $I G U A L$}

Igual posee «dos interpretaciones: una, comparativa, en que dos segmentos se relacionan por su semejanza y en que igual permanece dentro del ámbito de la construcción de la que forma parte; y otra, como marcador discursivo, ubicado en el margen izquierdo del segundo enunciado, en que vincula dos aserciones» (García Negroni y Marcovecchio 2013: 182). Tomando esta caracterización inicial, y con base en la NGLE (2009), García Negroni y Marcovecchio (2013), Fuentes (2011) y Hummel (2013), se discutirán los usos comparativos y discursivos de igual: los primeros reúnen la función de adjetivo y adverbio y los segundos, la función de marcador de discurso. Las investigaciones de corte cuantitativo-variacionista de San Martín (2004-2005, 2013 y 2016) analizan el uso de igual discursivo en el habla coloquial de Santiago de Chile y aportan datos para una visión dialectal acerca de esta función discursiva, por lo que resultan de sumo interés para

\footnotetext{
${ }^{3}$ De ahora en adelante PRESEEA-Caracas.
} 
la caracterización que se propone en estas líneas, si bien no se estudian en ellas los usos comparativos de igual ni el fenómeno de su diminutivo.

\subsection{Igual comparativo y pseudocomparativo}

En CONSTRUCCIONES COMPARATIVAS DE IGUALDAD, igual cumple la función de adjetivo y de adverbio. Cuando se trata de igual adjetivo, este aparece entre dos segmentos, con el significado 'de la misma naturaleza, cantidad o calidad de otra cosa', 'muy parecido o semejante' e indica que el primer elemento es de la misma naturaleza, parecido o semejante al segundo elemento $^{4}$. Es el significado que expresa el ejemplo (4):

(4) La lechuza no es igual que el búho

En ejemplos como (5), igual se emplea con el significado de 'de la misma manera', 'del mismo modo', esto es, con valor adverbial.

(5) Se apellida igual que yo

En los casos adverbiales el complemento solo puede aparecer introducido por la conjunción que (6a); pero, si cumple la función adjetival, el complemento puede alternar con la preposición y con el complemento conjuntivo, como en (6b):

(6) a. Trabajan igual que tú $\sim *$ Trabajan igual a ti

b. Es igual que tú $\sim$ Es igual a ti

Igual adverbial cuantifica a adjetivos (Era igual de guapo que su abuelo) y a adverbios (Cantas igual de bien que un profesional), como se señala en el Manual de la NGLE (RAE 2010). En el PRESEEA-Caracas se registran los usos de igual en construcciones comparativas: igual adjetivo se muestra en (7) e igual adverbio, en (8):

(7) I: aquí se llama el rabipelado / en Oriente no se llama así / allá se llama yaguare/

E: ¿yaguare?/

I: si allá en Oriente se llama yaguare / el puercoespín aquí es [igual] allá / también se llama puercoespín / eso sí es rico/ (CARA_H12_040)

(8) I: fue lo que pasó con Mérida / Mérida yo fui la primera vez / y después fui tres veces más seguidas / yo no sé por qué pero fui tres veces $<$ risas = "todos" $/>$ me está pasando con Margarita [igual] ¿no? / ahora agarro la excusa de que alguien me diga (CARA_H23_087)

\footnotetext{
${ }^{4}$ Igual también aparece en las locuciones al igual que, dar igual, de igual a igual, por igual, sin igual, etc. (cf. DILE 2001).
} 
Al tratarse de construcciones comparativas de igualdad, la comparación que se establece puede referirse a individuos, situaciones o estado de cosas, de acuerdo con la NGLE (RAE y ASALE 2009).

\subsection{Igual discursivo ${ }^{5}$}

Ahora bien, hay casos en los que no es posible hacer una interpretación de igualdad; son las CONSTRUCCIONES PSEUDOCOMPARATIVAS o falsas comparativas (cf. Sáez del Álamo 1998), que corresponden a la extensión de igual a las funciones discursivas, como se aprecia en los ejemplos que ofrece Sáez del Álamo (1998: 1179):

(9) a. Teo (igual/lo mismo) canta que baila, pero mucho más canta

b. Yo (igual/lo mismo) como carne que pescado, pero mucho más carne c. (Igual/lo mismo) voy al cine que al fútbol pero más al cine

A partir de los usos comparativos, igual experimenta un desplazamiento hacia los usos discursivos, a «lecturas divergentes», en palabras de García Negroni y Marcovecchio (2013). Bien podría considerarse la existencia de una macrofunción discursiva que se bifurca en dos subfunciones: una primera, en la que igual funciona como marcador de modalidad epistémica, y una segunda subfunción, en la que igual funciona como marcador de reformulación no parafrástico de distanciamiento. El tránsito semánticopragmático que lleva de la función comparativa a la discursiva se desarrolla en un continuo de gramaticalización en el que «es clave el contenido de cuantificación implícito en igual, que hace posible que se desprendan diferentes orientaciones argumentativas» (García y Marcovecchio 2013: 167). Del mismo modo, Fuentes (2011) afirma que se trata de un proceso de gramaticalización que acarrea pérdida de valor léxico y el desarrollo de valores modales.

El paso de funciones sintácticas a funciones de discurso justifica, para Martí Sánchez (2008), que igual se considere un operador pragmático, pues ejerce «funciones relacionadas con la (inter) subjetividad y modalidad lingüísticas dentro de una posición marginal» (2008: 80). Así, las subfunciones de modalidad y de reformulación de igual, en nuestra opinión, corresponderían a su condición de operador argumentativo. También Hummel (2013) ha estudiado los usos de igual a la luz de los conceptos de polisemia y polifuncionalidad de los marcadores del discurso, esto es, distintas funciones, distintas formas de igual, como se aprecia en la tabla 1, en la que autorresume dichas formas.

\footnotetext{
${ }^{5}$ Utilizaremos la locución marcador de discurso en su sentido más general para referirnos a las funciones epistémica y de distanciamiento.
} 
TABLa 1. Polisemia y polifuncionalidad del español igual

(Hummel 2013: 65)

\begin{tabular}{lll}
\cline { 2 - 3 } & Conceptual meaning & \multicolumn{1}{c}{ Function } \\
\hline Igual $_{1}$ & Equal & Adjective \\
\hline Igual $_{2}$ & In the same way & Adverb of manner \\
\hline Igual $_{3}$ & Also & Adverb with inclusive function \\
\hline Igual $_{4}$ & Perhaps & Epistemic adverb \\
\hline Igual $_{5}$ & In spite of something & Adversative-concessive connector \\
\hline
\end{tabular}

Así como Fuentes (2011) y García Negroni y Marcovecchio (2013), Hummel (2013) propone una secuencia evolutiva de igual discursivo que parte del significado básico (de adverbio de manera) y avanza hacia los significados «emergentes» also 'también', perhaps 'quizás', in spite of something 'a pesar de algo'.

\subsubsection{Igual epistémico}

En cuanto a la función discursiva, como se ve en (10), igual se puede emplear como adverbio de duda, sustituible por quizás, a lo mejor. No se trata de una construcción comparativa de igualdad, sino más bien de un enunciado en el que predomina la expresión de duda:

(10) coloq. quizá. Igual mañana nieva

El Diccionario de partículas discursivas (Briz, Pons y Portolés 2008) describe este valor de posibilidad o duda del siguiente modo: «Indica que alguien no tiene la seguridad suficiente para afirmar lo que dice. Por lo tanto, el miembro del discurso introducido por igual es una posibilidad». Para Fuentes, este igual expresa valores de hipótesis, duda, posibilidad, y afirma que ocurre un debilitamiento en el plano asertivo y el hablante muestra reserva de lo que afirma (Fuentes 2011: 85). Del mismo modo, Martín Zorraquino señala que el paso del adverbio comparativo al adverbio de modalidad epistémica se origina «como el resultado de un deslizamiento semántico a partir especialmente de una réplica en la que, con igual, se refuta la posibilidad de cumplimiento de un fenómeno, contraponiéndolo a su contrario» (2011: 40). Debido a este desplazamiento semántico, igual aparece al inicio del enunciado (igual te llamo) en el habla informal. 


\subsubsection{Igual concesivo}

En el continuo de gramaticalización, además del valor epistémico, igual expresa un valor concesivo que se interpreta como 'de todas maneras', 'de todos modos', 'de cualquier manera'; este valor representa la segunda lectura divergente de igual respecto de los usos primarios comparativos. Se trata de un operador de reformulación de distanciamiento gracias al cual el hablante expresa un distanciamiento de lo que ha afirmado, siguiendo a García Negroni y Marcovecchio (2013: 174). Documentados en el corpus, igual de distanciamiento también se da en el habla caraqueña:

(11) I: <entonces> todos tenemos horarios terribles / yo a veces tardo dos meses en hablar con mi hermana // porque ella está muy ocupada / E1: claro /

I: y yo también estoy muy ocupada / pero [igual] nos queremos / igualito // igualito / y nos hacemos falta igualito / y yo siento cuando hablo con ella / como si hubiese hablado ayer / así hayan pasado / dos o tres meses /

E1: eso / eso es chévere (CARA_M23_094)

En este ejemplo, el segmento que sigue a igual es un segmento antiorientado que expresa un contenido contrario al que se espera del segmento anterior y yo también estoy muy ocupada, anunciado por pero. Señala Fuentes que en estos usos hay «debilitamiento en la aserción, frente al valor aditivo propio de su otro uso como conector y como comparativo. Exige un contexto antiorientado, y aparece entre pausas, al inicio del enunciado, en la distribución típica de un conector» (2011: 91). Martín Zorraquino y Portolés consideran de todas maneras, de todos modos, como marcadores de reformulación de distanciamiento: «su significado nos obliga a concluir que ni esta manera que presenta el primer miembro, ni todas las demás posibles, impiden la conclusión que se mantiene en la reformulación» (1999: 4132).

\subsubsection{Igual de adición}

Fuentes describe un conjunto de variaciones que cabalgan entre la expresión de igualdad y la expresión discursiva y que denomina 'uso de cohesión por deixis con lo anterior'; estos usos se distinguen porque igual ha perdido el significado comparativo y sirve como conector de adición, especialmente con verbos como ocurrir, pasar, suceder, acontecer ${ }^{6}$. En el uso

\footnotetext{
${ }^{6}$ El ejemplo, que ofrece la autora, ilustra este uso: «La fibra, por ejemplo, en la actualidad aún eliminada de muchos alimentos, curiosamente se añade a otros porque se ha demostrado que es una sustancia indispensable para el buen funcionamiento del organismo huma-
} 
aditivo, igual alterna con también; se ubica entre dos enunciados y puede combinarse además con conjunciones (pero igual...). En (12) puede apreciarse cómo igual está más cerca del valor aditivo que del comparativo:

(12) I: <cita> vamos a hacer esto / pa' ayudar a mi papá / vamos a hacer lo otro / vamos a hacer esto </cita $>$ / pero cuando estábamos más pequeños mi papá tiene que haber pasado bastante <alargamiento/> trabajo allá en el monte / mi mamá [igual] <silencio/> pero nos criamos // por lo menos nos criaron / fue bastante $<$ silencio/ $>$ (CARA_H31_029)

Por otra parte, los datos que aporta el estudio diacrónico de Fernández Alcaide son esenciales para comprender la gramaticalización de igual. En (2011b), la autora examina datos extraídos del CORDE (siglos XVI-XX) para constatar la evolución de los usos de igual como sustantivo, de igualmente y de igual como adverbio, y encuentra que los usos adverbiales se consolidan a partir de la segunda mitad del siglo xx, período en el que se desarrollan los usos modales hasta la actualidad (cf. también Suárez Hernández 2017).

Con un enfoque sociolingüístico, San Martín (2004-2005) analizó la función pragmática y la estratificación social de igual como marcador de discurso en el habla de Santiago de Chile. Se trata de un estudio variacionista con una muestra de 72 entrevistas, en el que el autor considera, además, tres factores sociales: edad, sexo y nivel de estudios. Para el análisis, San Martín define la variable lingüística 'reformulador de distanciamiento' con tres variantes: igual, en todo caso y de todas maneras. Una vez identificados los casos de cada uno de los marcadores, los resultados muestran que igual es el más frecuente de los tres con una frecuencia muy alta de 96,2\% de un total de 554 casos; por su parte, de todas maneras apareció 17 veces (3\%) y en todo caso solo 4 veces, esto es, el 0,8\%. De la influencia de los factores sociales hay que destacar que la edad, cuyo examen arroja resultados diferenciadores, es el más relevante, pues el $82 \%$ de los casos de igual corresponden a los más jóvenes de la muestra, mientras que los de edades intermedias alcanzan el 13,5\% de los casos y los de más edad el 4,5\%. Al examinar el papel del sexo, el autor encuentra que hay más casos de igual de distanciamiento entre las mujeres, el $54 \%$, frente al $46 \%$ de los hombres. Por último, el nivel socioeconómico muestra una distribución algo relevante, pues el 41,5\% de los casos corresponden a los hablantes de estrato social medio. Con base en el análisis del tiempo aparente, San Martín concluye que los usos discursivos de igual representan un cambio lingüísti-

no. Igual ocurre con ciertos minerales y vitaminas que se eliminan porque interesa facilitar su transporte a largas distancias, conservación por periodos más dilatados de lo normal o para mejorar su aspecto visual» (Revista Natural 45/3, 2003, España, CREA) (Fuentes 2011: 80). 
co en marcha en la comunidad de Santiago de Chile. En un estudio posterior (2013), San Martín amplia el grupo de marcadores al incorporar de todas formas con el fin de profundizar los resultados de su estudio anterior. Con base en el análisis de 54 entrevistas, el autor confirma que igual es el marcador más empleado con un $97,8 \%$ de ocurrencias, frente a solo 7 casos de de todas maneras (1,2\%), 6 de en todo caso $(0,9 \%)$ y 1 caso de todas formas $(0,1 \%)$. Al analizar la incidencia de los factores sociales, la edad, de nuevo, es el más importante pues son los hablantes más jóvenes quienes emplean igual discursivo (56,5\% de los casos). Este hallazgo lleva a San Martín a ratificar su propuesta de que se trata de un cambio lingüístico en marcha. Finalmente, en (2016), este autor amplía a 120 entrevistas la muestra de estudio y llega a las mismas conclusiones: igual es el marcador de reformulador de distanciamiento más empleado por los hablantes santiaguinos, con un $95 \%$ de las ocurrencias (1300/1356), frente a un 2,3\% de en todo caso y un $1,9 \%$ de de todas maneras. Respecto de los factores sociales, el autor concluye que ni el sexo ni el nivel socioeconómico y la edad son significativos estadísticamente en el fenómeno pero que el empleo de igual discurso es más frecuente entre los hablantes jóvenes (2016: 274).

Las investigaciones de San Martín sobre los usos discursivos de igual, además de su análisis como un fenómeno de cambio lingüístico en marcha en el habla chilena, confirman la distribución geolectal de esta función de igual. $\mathrm{Al}$ respecto, el DPD aclara:

En la lengua coloquial de España se usa también este adverbio, seguido de un verbo en indicativo, con el significado de 'a lo mejor, posiblemente': "Si cada vez que llamen a la puerta te vas a poner así, igual acabas mala del corazón» (Mtn Gaite Fragmentos [Esp. 1976]); «Tu hermana igual necesita ayuda» (Vallejo Hölderlin [Esp. 1984])

En la misma línea, el Diccionario de Americanismos (2010) reporta el uso de igual concesivo ('a pesar de todo', 'no obstante') como propio en Bolivia, Argentina, Uruguay, Ecuador, Chile, Paraguay, Colombia, Panamá, Cuba y República Dominicana. Los usos discursivos de igual se bifurcan: la interpretación de marcador de reformulación no parafrástico de distanciamiento es común en las variedades americanas, mientras que en las variedades peninsulares predomina igual con valor epistémico ${ }^{7}$. En síntesis,

7 García Negroni y Marcovecchio comentan la siguiente situación comunicativa: «La experiencia personal de dirigirse a un auditorio de lingüistas peninsulares para tratar, entre otras cosas, el ítem igual como marcador discursivo de reformulación, dejó en evidencia que ese elemento dispara lecturas totalmente convencionalizadas, aunque irreconciliables, a un lado y otro del Atlántico. Así, una leyenda extraída de las vidrieras de un comercio del norte de la Argentina: A las mujeres, no se las golpea. Igual, no entienden, que en algunas variedades hispanoamericanas, por lo menos, provoca la interpretación de igual como un marcador de 
el valor concesivo se registra en los dialectos americanos y el valor de modalidad se da en los peninsulares. Queda claro que igual es una partícula polisémica en un proceso de gramaticalización diferenciado en la diatopía del español.

\section{IGUAL + SUFIJO DIMINUTIVO}

Igual es una base bisílaba, aguda, sin morfema de género, terminada en consonante, cuya estructura se modifica dependiendo de si la base cumple función adjetival, adverbial o discursiva, por lo que el diminutivo resultante puede concordar en género y número o mantenerse invariable en masculino singular. En la tabla 2 (página siguiente) se han ordenado las posibles variaciones morfológicas atendiendo a las funciones sintácticopragmáticas.

El diminutivo puede expresar atenuación así como también intensificación de la noción o cualidad: calentito significa 'muy caliente' y cerquita, 'muy cerca', por ejemplo.«En ahicito, allacito, igualito, mismito la intensificación se traduce en matices cercanos a los que expresan los adverbios justamente o exactamente», de acuerdo con RAE y AALE 2010: 169 (cf. también Lázaro Mora 1999). De acuerdo con Briz (1996), la intensificación es una «estrategia conversacional retórica para dar a entender más de lo que realmente se dice, de manipular realzando los enunciados con finalidades diferentes» (1996: 53). Albeida plantea en la misma línea:

La intensificación, desde un punto de vista semántico, es un valor de significado que se expresa lingüísticamente por medio de diversos procedimientos, fonéticos, morfológicos, sintácticos, etc. Supone una modificación de la cantidad y/o de la cualidad de un elemento del contenido proposicional del enunciado o un refuerzo de la aserción. A las formas lingüísticas que han adquirido entre sus componentes semánticos el valor de intensificación se les llamará intensificadores semánticos (2004: 54).

Como recurso intensificador, el diminutivo imprime mayor fuerza y realce a una cualidad y en el caso de igual, todas las formas con diminutivo resultantes intensifican la comparación de la noción comparada ${ }^{8}$ o el valor de duda o el distanciamiento de lo dicho, es decir, el valor discursivo. Ya que igual cumple funciones en el marco de la oración como también en el discurso, las formas en diminutivo se relacionan, por una parte, con el

reformulación no parafrástico de distanciamiento ( $\equiv$ 'de todas maneras / de cualquier manera'), generaba asombro e inquietud entre los españoles, dado que se les volvía totalmente opaca en tanto interpretaban igual como quizás / a lo mejor» (2013: 168).

${ }^{8}$ Por noción comparada debe comprenderse 'la naturaleza de lo que se compara'. 
TABLA 2. Variaciones pragmalingüísticas del diminutivo de igual

\begin{tabular}{|c|c|c|c|}
\hline & FunCIÓN & EJEMPLOS & $\begin{array}{l}\text { MORFOLOGÍA } \\
\text { DEL DIMINUTIVO }\end{array}$ \\
\hline \multirow{4}{*}{$\begin{array}{l}\text { Usos } \\
\text { comparativos }\end{array}$} & $\begin{array}{l}\text { Igual adjetivo } \\
\text { comparativo }\end{array}$ & $\begin{array}{l}\text { Luis es igual a Ana } \\
\text { Luis y Ana son iguales a Belkys }\end{array}$ & $\begin{array}{c}\text { Variable } \\
\text { igual/iguales (adjetivo } \\
\text { con flexión de número) }\end{array}$ \\
\hline & $\begin{array}{l}\text { Igualito adjetivo } \\
\text { comparativo }\end{array}$ & $\begin{array}{l}\text { Luis es igualito a Ana } \\
\text { Ana es igualita a Luis } \\
\text { Luis y Antonio son igualitos } \\
\text { a su papá } \\
\text { Ana y Bertha son igualitas } \\
\text { a su mamá }\end{array}$ & $\begin{array}{c}\text { Variable } \\
\text { igualita(s)/igualito(s) } \\
\text { (con flexión de número } \\
\text { y género) }\end{array}$ \\
\hline & $\begin{array}{l}\text { Igual adverbio } \\
\text { comparativo }\end{array}$ & $\begin{array}{l}\text { Se apellidan igual } \\
\text { que nosotros }\end{array}$ & \multirow{2}{*}{ Invariable } \\
\hline & $\begin{array}{l}\text { Igualito adverbio } \\
\text { comparativo }\end{array}$ & $\begin{array}{l}\text { Se apellidan igualito } \\
\text { que nosotros }\end{array}$ & \\
\hline \multirow{6}{*}{$\begin{array}{l}\text { Usos } \\
\text { discursivos }\end{array}$} & Igual epistémico & Igual llueve mañana & \multirow{6}{*}{ Invariable } \\
\hline & $\begin{array}{l}\text { Igualito } \\
\text { epistémico }\end{array}$ & Igualito llueve mañana & \\
\hline & $\begin{array}{l}\text { Igual } \\
\text { reformulador de } \\
\text { distanciamiento }\end{array}$ & $\begin{array}{l}\text { Estamos muy ocupadas, } \\
\text { pero igual nos queremos }\end{array}$ & \\
\hline & $\begin{array}{l}\text { Igualito } \\
\text { reformulador de } \\
\text { distanciamiento }\end{array}$ & $\begin{array}{l}\text { Estamos muy ocupadas, } \\
\text { pero igualito nos queremos }\end{array}$ & \\
\hline & $\begin{array}{l}\text { Igual conector } \\
\text { de adición }\end{array}$ & $\begin{array}{l}\text { Mi papá trabajó mucho, } \\
\text { mi mamá igual }\end{array}$ & \\
\hline & $\begin{array}{l}\text { Igualito conector } \\
\text { de adición }\end{array}$ & $\begin{array}{l}\text { Mi papá trabajó mucho, } \\
\text { mi mamá igualito }\end{array}$ & \\
\hline
\end{tabular}

proceso de gramaticalización de igual y, por el otro, con el proceso de gramaticalización del diminutivo por subjetivización. A partir de su estudio comparado del diminutivo en distintos dialectos del español, Reynoso $(2003,2005)^{9}$ propuso que el diminutivo experimenta un proceso de gra-

${ }^{9}$ En dicha investigación, la más amplia y multidialectal de los estudios sincrónicos sobre el diminutivo con corpus contemporáneos, la autora mexicana analizó cuantitativa y cualitativamente un corpus multidialectal, compuesto por muestras orales y escritas, pertenecien- 
maticalización por subjetivización. La autora mexicana analiza la alta frecuencia de uso de diminutivos, la influencia de las lenguas de sustrato y de registro, y las funciones referenciales y pragmáticas con las que se emplea el sufijo apreciativo en cada una de las variedades estudiadas. Para Reynoso,

[...] las marcas de disminución parecen encapsular una diversidad de significados que se relacionan entre sí y de múltiples maneras con todos los elementos del acto comunicativo. De esta manera, como muestra el corpus analizado, el diminutivo parece ser una flexible herramienta pragmática de la lengua española que sirve para marcar líneas de significación que el hablante necesita en cada escena discursiva. La naturaleza pragmática del diminutivo explica la esencia polisémica de la marcación en términos de las necesidades comunicativas del hablante. Las marcas de disminución en español parecen cubrir un amplio espectro de necesidades pragmáticas de comunicación en esta lengua. (Reynoso 2005: 85)

La gramaticalización por subjetivización del diminutivo ${ }^{10}$ permite aunar la descripción de los cambios morfológicos que ocasiona el sufijo en la forma base y la variedad de significados subjetivos que expresa al codificar el afecto, el respeto, la descalificación, en detrimento de los valores referenciales, relacionados con la dimensión del tamaño. En este sentido, igualito(s)/igualita(s) representan nuevas formas, polifuncionales, subjetivas, de igual, como adjetivo-adverbio y, además, muestran la extensión del diminutivo a nivel del discurso, fenómeno que no ocurre con otros adverbios diminutivizados, por ejemplo tempranito o ahorita, ni en los otros dialectos americanos en los que se emplea igual con valores epistémicos.

\section{Metodología}

Se identificaron todos los casos de construcciones comparativas/discursivas con igual/iguales y sus variantes en diminutivo (igualita/as/, igua-

\footnotetext{
tes tanto a muestras urbanas como rurales del español americano y peninsular. Reynoso se planteó «la comparación sincrónica de la marca de diminutivo en el español de cuatro zonas dialectales, dos de ellas caracterizadas por su contacto con lenguas y culturas de sustrato: 1) Altiplano central, costa pacífica, costa atlántica de México y 2) la zona andina; y otras dos cuyo desarrollo histórico-cultural no sólo carece de culturas de sustrato y adstrato, sino que es totalmente divergente respecto de las dos primeras: 3) Madrid, España y 4) Buenos Aires, Argentina» (2003: 18).

${ }^{10}$ De acuerdo con esta autora, «la gramaticalización consiste en la fijación o rutinización de estrategias discursivas. Esto es, una forma o construcción que en un estado de lengua dado opera en un nivel pragmático o discursivo y codifica significados pragmáticos o particulares, se vuelve con el paso del tiempo una estructura convencional, carente ya de condicionamientos pragmáticos» (2014: 332).
} 
lito/os) en las 108 entrevistas semiformales, estratificadas por edad, sexo y grado de instrucción que integran el PRESEEA-Caracas 2004-2010 ${ }^{11}$ y, seguidamente, se clasificaron los casos según las funciones. En segundo lugar, con base en las frecuencias absolutas y relativas de la forma base y las formas diminutivizadas, se analiza la distribución del uso del diminutivo de acuerdo con los factores sociales considerados en el corpus. No hay, hasta donde se ha podido constatar, estudios sobre el diminutivo de igual, por lo que no hay antecedentes directos, pero en la sección de resultados se compararán, de modo general, algunos datos de las investigaciones de San Martín. A continuación se presentan los datos del habla caraqueña.

\section{Discusión}

\subsection{Resultados generales}

De las 508 construcciones identificadas, hay que destacar que la función comparativa predomina en un $88,7 \%$ de los casos $(450 / 508)$, mientras que la función discursiva alcanza un 11,3\% (tabla 3); como se aprecia en el gráfico 1, el diminutivo registra 194 ocurrencias, un $38,2 \%$ de casos, frente al $61,8 \%$ de la forma base.

TABLA 3. Funciones del diminutivo e igual según la función en el PRESEEA-Caracas

\begin{tabular}{|c|c|c|c|c|}
\hline & & Comparativa & Discursiva & Total \\
\hline \multirow[t]{2}{*}{ Igualitos/s/ita/s } & & 166 & 28 & 194 \\
\hline & $\%$ & 37 & 49 & 38 \\
\hline \multirow[t]{2}{*}{ Iguales } & & 284 & 30 & 314 \\
\hline & $\%$ & 63 & 51 & 62 \\
\hline \multirow[t]{2}{*}{ Total } & & 450 & 57 & 508 \\
\hline & $\%$ & 88,7 & 11,3 & \\
\hline
\end{tabular}

${ }^{11}$ El PRESEEA-Caracas 2004-2010 forma parte del proyecto panhispánico Proyecto para el estudio sociolingüístico del español de España y América (cf. Moreno Fernández 2005, 2006, 2016). Para la metodología general del proyecto coordinado, puede consultarse el portal digital <http://preseea.linguas.net/>, y para la descripción del corpus caraqueño remito a Bentivoglio y Malaver (2012). 
GráfICO 1. Casos de Diminutivo/No diminutivo

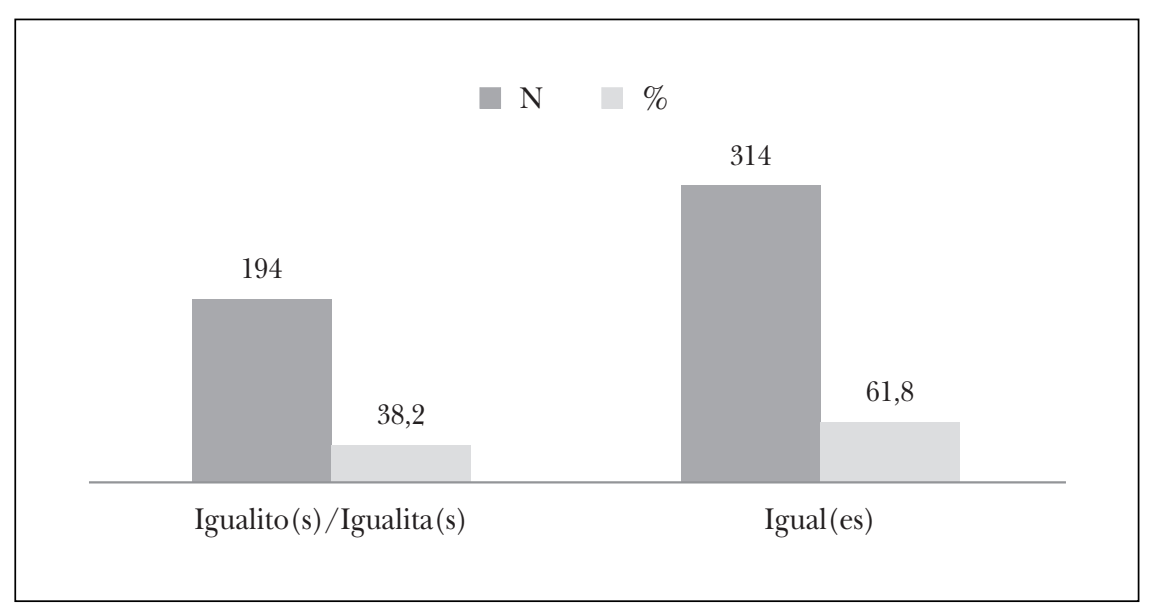

Interesa también, en segundo lugar, que el $63 \%$ de la función comparativa es realizada con igual, como también se indica en la tabla 3; sin embargo, la función discursiva se comporta de forma distinta ya que el diminutivo aumenta, alcanzando el $49 \%$ de los casos, por lo que se aprecia que abarca tanto los usos comparativos como los discursivos, mientras que igual abarca, principalmente, los primeros. Este hallazgo se relaciona con la extensión del diminutivo adjetival/adverbial a los usos epistémicos o modales, principalmente de igualito, forma masculina singular que constituye el $88 \%$ de los casos de diminutivos, dato que se presenta en la tabla 4 a continuación.

\begin{tabular}{|c|c|c|}
\hline & $\mathrm{N}$ & $\%$ \\
\hline Igualito & 170 & 88 \\
\hline Igualitas & 8 & 4 \\
\hline Igualita & 6 & 3 \\
\hline Igualitos & 10 & 5 \\
\hline Total & 194 & \\
\hline
\end{tabular}

La diferencia entre los 170 casos de igualito y los 24 restantes, distribuidos en 10 casos de igualitos $(5 \%)$ y 14 casos de la forma femenina, 8 en plural y 6 en singular, $4 \%$ y $3 \%$, respectivamente, sugiere que es la forma 
GRÁFICO 2. Funciones de igualito en el PRESEEA-Caracas

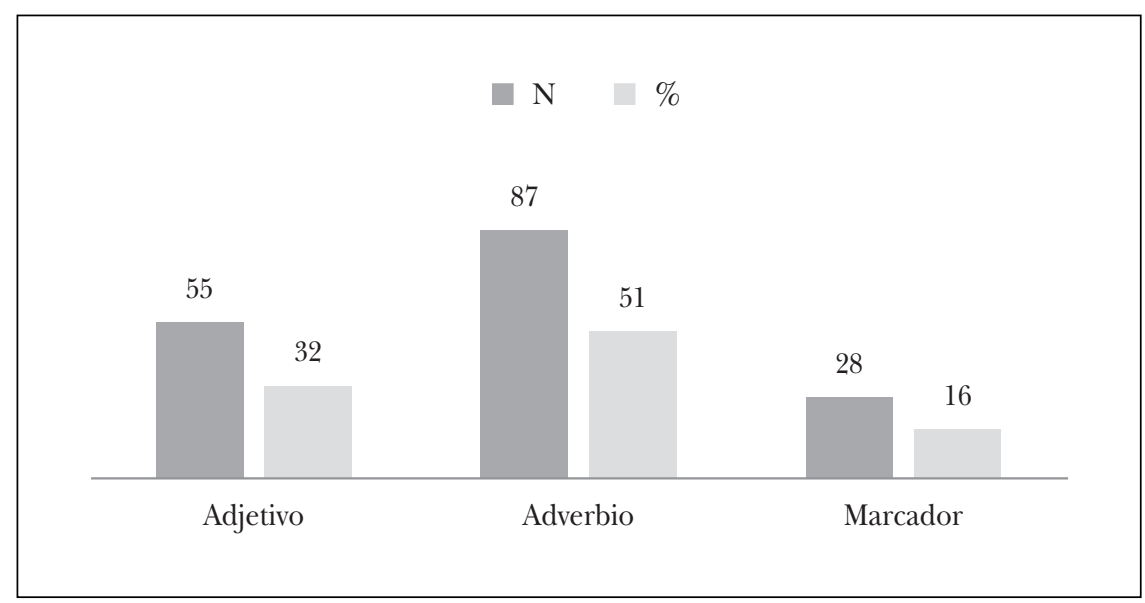

masculina singular la que se extiende; la frecuencia tan alta de igualito encuentra su explicación -como se ve en el gráfico 2- en que se emplea en todas las funciones de igual: $32 \%$ como comparativo adjetival, $51 \%$ como comparativo adverbial y $16 \%$ como marcador discursivo.

Respecto de sus usos adjetivales, en los ejemplos que siguen aparece igualito con el significado de 'la misma naturaleza, cantidad o calidad de otra cosa', 'muy parecido o semejante':

(13) I: es [igualito] // en el servicio militar cuando tú eres nuevo / te comes las verdes (CARA_H11_004)

(14) I: pero es [igualito] a mi abuela $</$ cita $>$ o sea / a la abuela de él / (CARA_H12_040)

(15) I: lo hubiese conocido en otro ambiente / y hubiese visto cómo en verdad viven los de Río / pero / eeh / Sao Paulo sí / es [igualito] que Caracas (CARA_H23_088)

(16) I: ¿ves? ese es el problema justamente ¿ves? / entonces esa parte didáctica es fundamental y en Historia y Geografía es [igualito] (CARA_H33_101)

Los usos adverbiales se ilustran en (17) al (19) y en ellos el diminutivo cumple la función comparativa con el significado de 'de la misma manera', 'del mismo modo':

(17) I: ella trabajaba [igualito] / de siete a siete / y yo trabajaba de siete a siete [igualito] (CARA_H23_089) 
(18) I: <cita $>$ esto te va a costar un ojo de la cara $</$ cita $>$ te cuesta [igualito] que cualquier / eeh / cosa de Europa pero (CARA_H23_088)

(19) I: los musulmanes / allá están los santeros / y todos creemos en Dios / Dios es un pollo / tú lo muerdes por aquí / tú lo muerdes por allá / pero él te va a querer [igualito] / como quiere / a todos ellos porque (CARA_H21_016)

En los usos discursivos de igualito, como (20), ya no hay expresión alguna de comparación:

(20) I: ya lo vi todo / eso es un trauma que tiene él / no sabe nadar entonces/ ya del tamañote que tiene imagínate / nos pasa a nosotros tres / ese parece una varita e pulla loco/[igualito] le da miedo el agua (CARA_M12_048)

Se aprecia que el segmento discursivo que sigue a igualito expresa una reformulación de lo que se viene diciendo e introduce una contraorientación argumentativa; son los usos de reformulación por distanciamiento, en los que el diminutivo podría sustituirse por 'de todos modos', 'de todas maneras'. Fueron frecuentes en el corpus los casos de pero + igualito en este contexto de marcador de distanciamiento:

(21) I: no pero [igualito] tienes que darme tu cédula de $</$ cita $>/<$ cita $>$ / toma mi cédula $</$ cita $><$ risas = "E2" $/>/$ (CARA_M12_047)

(22) E1: pero / pero tienes que empezar a estudiar / lo dejé para hacer deporte /

I: exacto /

E1: es un buen proyecto / Roosevelt / eso de /

I: sí / es buen proyecto / pero [igualito] estudio lo que me gusta y entreno a los muchachos / normal / (CARA_H12_041)

(23) I: después del accidente él quedó sentado / o sea se < vacilación/> sentaba a veces / lo paraban y el caminaba solo / pero cuando yo bajaba del colegio él me veía feo / y me daba miedo pero [igualito] no le demostraba el miedo sino que seguía (CARA_M12_048)

La combinación con otro marcador, propia de igual discursivo, se encontró también en el corpus caraqueño:

(24) I: y yo también estoy muy ocupada / pero igual nos queremos / (CARA_M23_094)

(25) I: pero [igual] le pagan demasiado / (CARA_H22_054)

Este resultado coincide con lo que señala San Martín (2003-2004: 220) sobre la predisposición de igual-marcador de combinarse con pero, o sea y porque en el habla chilena. 
En cuanto a las otras variantes del diminutivo, igualita se emplea únicamente como adjetivo (6 casos) e igualitas como adjetivo y adverbio, 4 veces cada uno. Con función adjetival se documentan los ejemplos siguientes en el corpus caraqueño:

(26) E2: ¿cómo era Europa? /

I: [igualita] / bella y grande como siempre / bueno es una maravilla // solamente la parte histórica y artística (CARA_H32_065)

(27) I: la casa está [igualita] / ahí vivimos nosotros muchos años / (CARA_H33_099)

(28) I: la puerta y las niñas se ponían de pie / todas derechitas / las faldas [igualitas] todas (CARA_H33_101)

(29) I: <cita> coño / las patinetas estas $</$ cita / patinetas de tres ruedas / [igualitas] a los monopatines estos / solo que eran de tres ruedas (CARA_H23_089)

Se puede constatar que la forma diminutivizada establece relaciones de concordancia de género, como en (26) y (27), y de número, como en (28) y (29). Los ejemplos de igualitos adjetival (9 casos) aparecen con los verbos ser y estar, como en (30) y (31):

(30) I: los muchachos estudiantes también están [igualitos] (CARA_H32_061)

(31) I: ellos son [igualitos] / bueno / ellos ya tenían / ellos han tenido (CARA_H21_018)

Y en el caso de función adverbial se encuentran ejemplos en los que el diminutivo hace concordancia, además de número plural, de género:

(32) I: pero bueno / ahí vamos <risas = "I" / / / entonces las relaciones eh / con / con Dios / bueno / van [igualitas] / (CARA_M31_033)

(33) I: porque todas habíamos estudiado [igualitas] (CARA_M23_095)

(34) I: o sea que fueron casi todos los bisnietos y los / todas las niñitas las vestimos [igualitas] (CARA_M33_108)

(35) E1: ¿¿íi? o sea ¿usted tiene unas trillizas? /

I: trillizas gemelas / se parecen [igualitas] (CARA_H32_064)

(36) I: en cambio los morochos sí se parecen / tú los ves a los dos [igualitos] / tú dices / (CARA_H21_018)

No se encontraron casos de igualito ni igual con valor epistémico y solo tres ejemplos de la locución verbal dar igual ${ }^{12}$.

${ }^{12}$ Se identificaron 21 casos de igualmente. 


\subsection{Alternancia e intensificación}

El valor intensificador del diminutivo queda plasmado cuando el hablante alterna en un mismo segmento discursivo con la forma base; el habla caraqueña registra casos en los que el hablante emplea igual adjetivo y luego recurre al diminutivo en una estrategia de intensificación que desarrolla para resaltar el sentido de su opinión:

(37) tan aburrido / o sea / o<alargamiento/> vas pa' / vas pa' Mayami / y es igual que ir para<alargamiento/> Chicago / Kentucky / porque todo tiene / un McDonalds / un Wendy's // un no sé qué / y las calles son iguales / y la gente es [igual] / en todos lados // unos más negros / otros más blancos // pero todo es [igualito] / las tiendas los <extranjero $>$ malls $</$ extranjero $>$ todo todo / todo es igualito / además con ese rollo que te niegan la visa / (CARA_M23_094)

(38) I: siempre es bueno <ruido = "chasquido boca" $/>$ no quedarse callado para nada / si todos somos hermanos / si todos somos [iguales] / independientemente de que tengamos o no tengamos $<$ ruido $=$ "silbido" / somos / [igualitos] / ¿me entiendes? // (CARA_M22_056)

En un caso como (39), el diminutivo intensifica nuevamente el uso discursivo de la partícula base:

(39) que tu jefe te lo firmara / y entonces tú dejarlo en recepción para que tú salieras / o sea / y por Dios si tú llegabas tarde tenías que ir corriendo $<$ risas = "I" / > a marcar el carnet y no llegar muy tarde y bueno / para salirse [igual] / o sea / [igualito] / a las horas del mediodía (CARA_M13_081)

Hasta aquí, los usos analizados del diminutivo abarcan la intensificación de igual en el marco de la oración, en sus usos comparativos adjetivales y adverbiales, y se extienden al uso a nivel del discurso, extraoracional.

\subsection{Los factores sociales}

Los porcentajes de uso del diminutivo de acuerdo con las dimensiones sociales presentan diferencias que merecen destacarse.

De acuerdo con los datos del gráfico 3, las mujeres emplean más tanto la forma base como el diminutivo; y en el gráfico 4 se aprecia que los hablantes de edades intermedias tienen bastante más casos de diminutivo que los hablantes de los grupos etarios extremos: 48,3\% frente al 35,1\% de los más jóvenes y 33,9\% de los mayores, entre los que se registran menos diminutivos en todo el corpus analizado. 
GrÁfICO 3. Distribución de igual $e$ igualito(s)/ita(s) en el PRESEEA-Caracas según el sexo (\%)

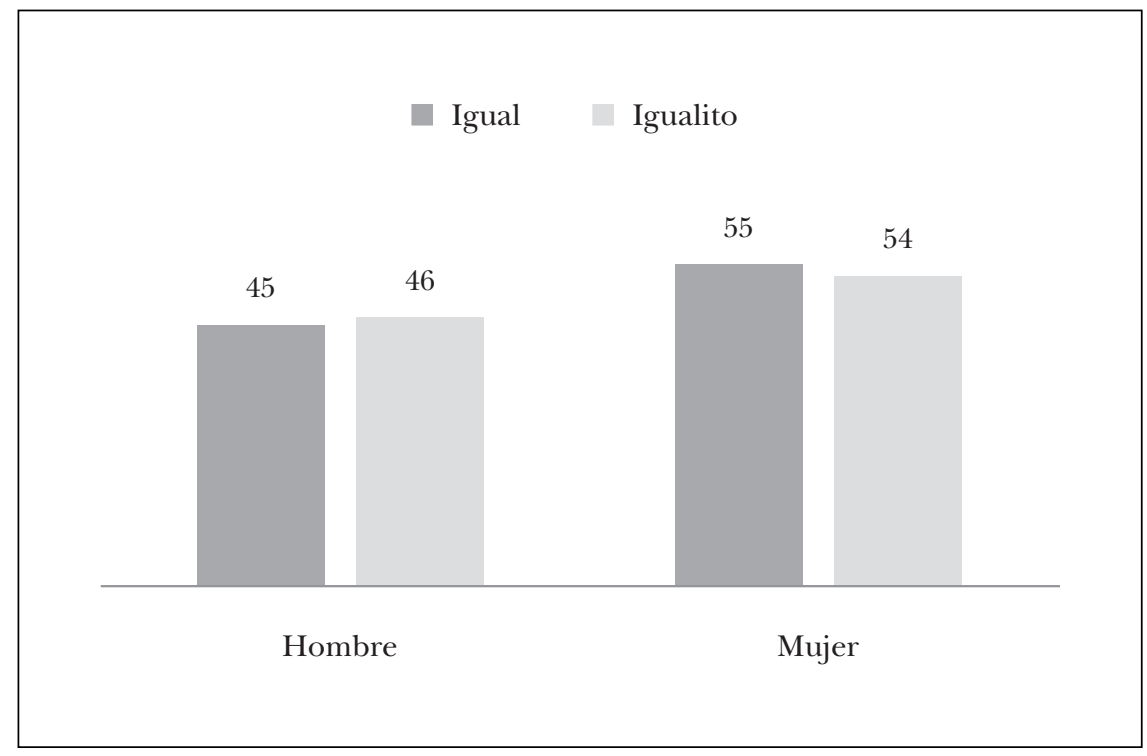

GráfICO 4. Distribución de igual $e$ igualito(s)/ita(s) en el PRESEEA-Caracas según la edad

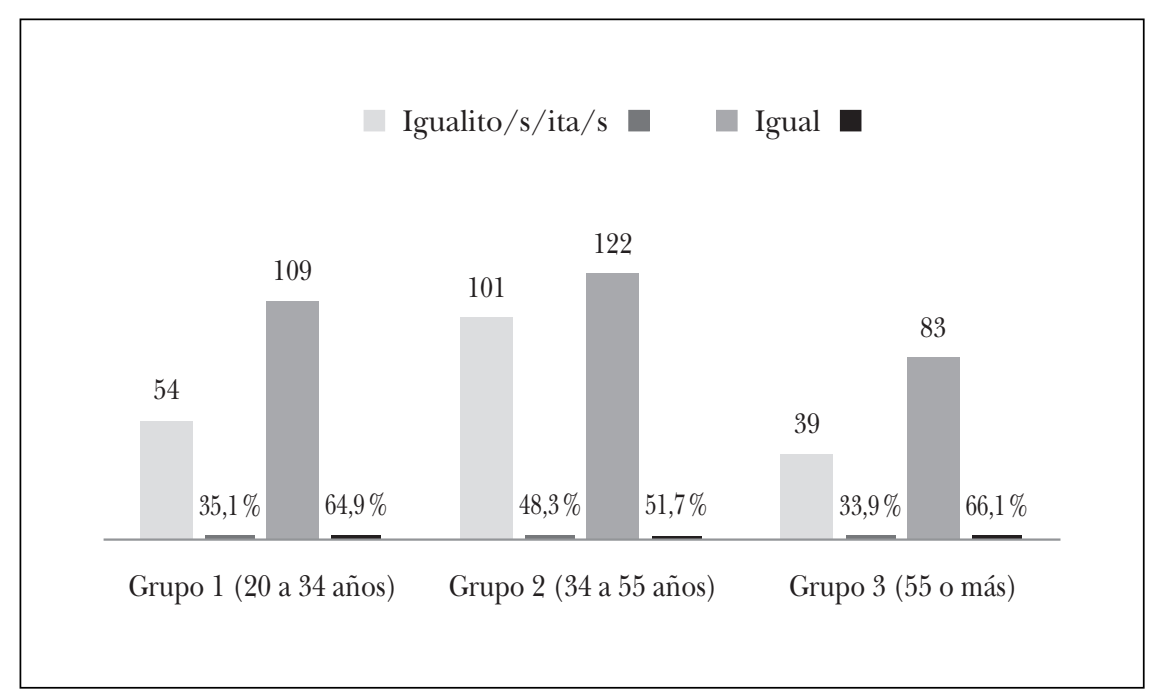


En cuanto al grado de instrucción, los datos que se presentan en el gráfico 5 señalan que el 43,3\% de los casos de diminutivo se concentran entre los hablantes de estudios secundarios y, por el contrario, los hablantes más instruidos son los que menos los emplean. En cuanto a igual, su empleo se da en proporciones similares entre los tres grupos etarios.

GrÁFICO 5. Distribución de igual $e$ igualito(s)/ita(s) en el PRESEEA-Caracas según el grado de instrucción

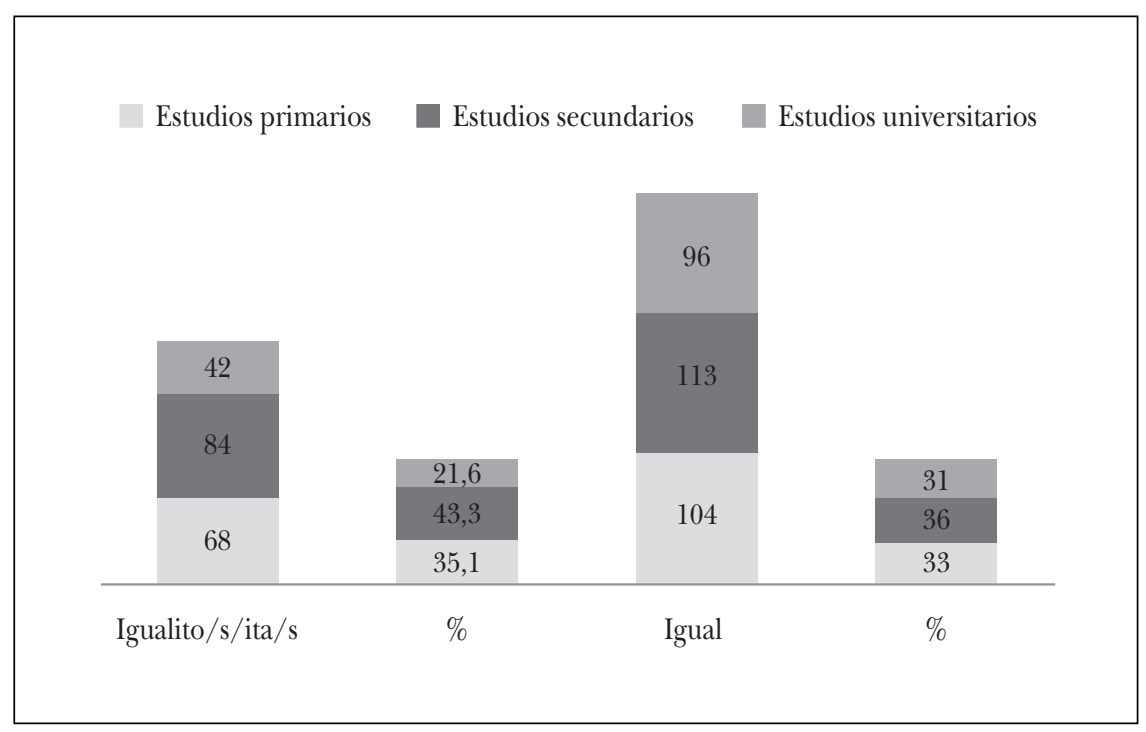

Estos resultados del sexo, edad e instrucción no muestran una marcada diferenciación social; a diferencia de los resultados de San Martín (20032004, 2016) para el habla de Santiago de Chile, ni el sexo ni la edad alcanzan diferencias marcadas en Caracas; no obstante, sí permiten confirmar que en este dialecto se emplea igual como reformulador de distanciamiento -como en Argentina y Chile- y que alterna con el diminutivo igualito, el cual contribuye en el proceso de gramaticalización de igual como marcador de discurso.

\section{Conclusiones}

Los hallazgos identificados confirman que el habla caraqueña se distingue por el uso de diminutivos adjetivales y adverbiales. Basados en los datos del PRESEEA-Caracas, se puede concluir, en primer lugar, que: 
1) El diminutivo aparece un $38 \%$ en los contextos de igual, lo que constituye un estado de alternancia cuya explicación se halla en el deseo del hablante de intensificar las nociones de igualdad, por una parte, y de distanciamiento, por la otra.

2) El diminutivo se comporta, al igual que la forma base, de manera polisémica, cumpliendo funciones de adjetivo, adverbio y marcador de reformulador de distanciamiento.

3) La función principal de igual es la comparativa, bien como adjetivo o como adverbio, contextos en los que hay alternancia con el diminutivo, pero en el caso de la función discursiva, igual desciende su frecuencia y el diminutivo asciende a un $49 \%$ de los casos. Hay que llamar la atención sobre este resultado ya que puede indicar una especialización funcional del diminutivo.

4) La forma masculina singular, igualito, es la de mayor frecuencia en el corpus.

En segundo lugar, en cuanto a los cambios morfológicos que experimenta igual cuando el sufijo apreciativo se le añade (tabla 2), se deben destacar las diferencias entre la función adjetival y la adverbial. En la función adjetival, como cabe esperar, las formas del diminutivo concuerdan en género y número; en la función adverbial se constata que las formas plurales del diminutivo establecen concordancia de género y número, lo que muestra una modificación del paradigma de la forma base.

Los factores sociales, en tercer lugar, muestran cierta influencia en las elecciones de los hablantes: son las mujeres las más proclives a emplear el diminutivo y de edades intermedias, pero estos resultados no permiten hacer conclusiones absolutas sobre el modo en que las condiciones sociales determinan la elección del diminutivo, aunque las frecuencias relativas muestran diferencias que no deben dejar de ser consideradas en próximos estudios.

Por último, el empleo de igualito como marcador de reformulador de distanciamiento, por una parte, imprime singularidad al proceso de extensión del diminutivo en bases adverbiales en el habla caraqueña y, por la otra, representa una característica del proceso de gramaticalización de igual en esta variedad. 


\section{BIBLIOGRAFÍA}

\section{Diccionarios y obras de referencia}

Asociación de Academias de la Lengua EsPañola (2010): Diccionario de americanismos, Lima: Santillana Ediciones Generales.

Briz, Antonio, Salvador PONS y José PORTOLÉs (coords.) (2008): Diccionario de partículas discursivas del español. <www.dpde.es>.

Real Academia Española (2002): Diccionario de la lengua española. <http://www. rae.es>.

\section{Referencias bibliográficas}

AlbeldA, Marta (2005): La intensificación en el español coloquial, Tesis doctoral, Valencia: Universidad de Valencia.

Ambadiang, Théophile (1997): «Las bases morfológicas de la formación de diminutivos en español», Verba: Anuario galego de filoloxia 24, 99-132.

Bentivoglio, Paola e Irania Malaver (2012): «Corpus sociolingüístico de Caracas: PRESEEA-Caracas 2004-2010. Hablantes de instrucción superior», Boletín de Lingüistica 37-38, 144-180.

BRIZ, Antonio (1998): El español coloquial en la conversación. Esbozo de pragmagramática, Barcelona: Ariel.

Company Company, Concepción (2004): «Gramaticalización por subjetivización como prescindibilidad de la sintaxis», Nueva Revista de Filología Hispánica LII, $1-27$.

- (2014): «Residuos y paradojas en la diacronía de los adverbios en -mente. Evidencia para la gramaticalización», Nueva Revista de Filología Hispánica LXII, 329-356.

Fernández Alcaide, Marta (2011b): «Igual. Historia y valores (II): sustantivo y adverbio", Revista de Historia de la Lengua Española 6, 3-34.

Fuentes Rodríguez, Catalina (2011): «Conexión y debilitamiento asertivo: igual, igualmente, lo mismo». En Ramón González-Ruiz y Carmen Llamas (eds.), Gramática y discurso, Pamplona: EUNSA.

García Negroni, María Marta y Ana María Marcovecchio (2013): «No todo da lo mismo: de la comparación al distanciamiento. El caso de igual», Oralia 16, $167-186$.

Hummel, Martin (2013): «Polyfunctionality, polysemy, and rhetorical strategy. The functional, semantic and pragmatic motivation of discourse functions», Grazer Linguistische Studien 79, 63-91.

LÁzARo Mora, Fernando A. (1999): «La derivación apreciativa». En Ignacio Bosque y Violeta Demonte (dirs.), Gramática Descriptiva de la Lengua Española, Madrid: Espasa-Calpe, 4645-4682. 
MALAVER, Irania (2017): “"Ahorita”: Lexicalización y cambio lingüístico en la comunidad de habla caraqueña», Nueva Revista de Filología Hispánica 65/1, 27-57. $<$ http://nrfh.colmex.mx/index.php/nrfh/article/view/2828>.

MARTí SÁNCHEZ, Manuel (2008): «La hipótesis de la subjetivización en la pragmaticalización/gramaticalización de los operadores pragmáticos», Paremia 17, 79-90.

MARTín ZorRaQUino, María Antonia (2011): «Juan vendrá igual mañana (que no vino ayer)/Igual vendrá Juan Mañana». En María Victoria Escandell Vidal, Manuel Leonetti y María Cristina Sánchez (eds.), 60 problemas de gramática dedicados a Ignacio Bosque, Madrid: Akal, 400-405.

Martín Zorraquino, María Antonia y José Portolés (1999): «Los marcadores del discurso». En Ignacio Bosque y Violeta Demonte (coords.), Gramática descriptiva de la lengua española, Madrid: Espasa-Calpe, 4051-413.

Moreno Fernández, Francisco (2005): «Corpus para el estudio del español en su variación geográfica y social. El corpus "PRESEEA"», Oralia 8, 123-139.

- (2006): «Información básica sobre el Proyecto para el Estudio Sociolingüístico del Español de España y de América - PRESEEA (1996-2010)», Revista Española de Lingüistica XXXVI, 385-392.

- (2016): «En torno a PRESEEA: Notas de investigación y de sociología de la ciencia», Boletín de Filología 51/2, 369-376. <http://www.boletinfilologia.uchile.cl/ index.php/BDF/article/view/44883/46953>.

Real ACAdemia Española y Asociación de ACAdemias de la Lengua Española (2009): Nueva gramática de la lengua española. Morfología y sintaxis, Madrid: Espasa.

- y - (2010): Nueva gramática de la lengua española. Manual, Madrid: Espasa.

ReYNoso Noverón, Jeanett (2003): Los diminutivos en el español. Un estudio de dialectología comparada, Tesis de Doctorado, México: UNAM.

- (2005): «Procesos de gramaticalización por subjetivización. El uso del diminutivo en el español». En David Eddington (ed.), Selected Proceedings of the 7th Hispanic Linguistic Symposium, Somerville, MA: Cascadilla Proceeding Project, 79-86. <http://www.lingref.com/cpp/hls/7/paper1088.pdf >.

SÁEZ DEL Alamo, Luis Ángel (1998): «Los cuantificadores: las construcciones comparativas y superlativas». En Ignacio Bosque y Violeta Demonte (coords.), Gramática descriptiva de la lengua española, Madrid: Espasa-Calpe, 1129-1188.

SAN MARTín NúÑEZ, Abelardo (2004-2005): «Igual como marcador discursivo en el habla de Santiago de Chile: funciones pragmático-discursivas y estratificación social de su empleo», Boletín de Filología XL, 201-232.

- (2013): «Los reformuladores de distanciamiento en el corpus PRESEEA de Santiago de Chile», Boletín de Filología XLVIII, 171-199.

- (2016): «Los reformuladores de distanciamiento en el habla santiaguina: igual y sus equivalentes funcionales», Onomázein 34. <http://www.redalyc.org/articu lo.oa?id=134549291017>.

SuÁrez HernáNDeZ, Ariana (2017): Análisis diacrónico de adverbios con función discursiva: Hacia una descripción lexicográfica, San Millán de la Cogolla: Cilengua. 